\title{
La enseñanza basada en la indagación en el área de las ciencias experimentales
}

Francisco Javier Acosta Collazo

$\mathrm{E}$ n la actualidad, las prácticas pedagógicas presentan cierta complejidad al estar relacionadas con criterios de calidad externos, en donde los niveles de aprendizaje deberán ser evaluados y estar vinculados con el entorno. Además de lo anterior, en las ciencias experimentales como la Biología, se requiere de competencias disciplinares para la investigación en un entorno atractivo para el estudiante, en donde se promueva el desarrollo del pensamiento científico.

El enfoque basado en la indagación fomenta los cuestionamientos en lugar de las respuestas, de manera que, mediante la aplicación de una estrategia educativa denominada "investigación individual", se puede evaluar el proceso o ciclo de aprendizaje del método científico en los estudiantes. Como producto de la experiencia de aprendizaje, los jóvenes elaboran un "cartel científico" que refleja el desarrollo

del pensamiento crítico y creativo en una indagación estructurada.

En el ámbito de las propuestas didácticas para la educación científica de los jóvenes en el nivel internacional, la Organización de las Naciones Unidas para la Educación, la Ciencia y la Cultura (2005) hace énfasis en la necesidad de una alfabetización científica, no sólo en el sentido de formar futuros científicos, sino como parte de una educación general para los futuros ciudadanos; el aprendizaje de las ciencias es una actividad próxima a la investigación científica, sin embargo, es un reto en la educación del siglo XxI. Victoria Gutiérrez (1998), en una minuciosa investigación centrada en las actitudes de los estudiantes universitarios hacia la ciencia ante los índices de reprobación en el contexto de la Universidad Autónoma de Aguascalientes (UAA), concluye en la importancia de que el aprendizaje de los contenidos científicos esté en contacto con los problemas importantes en la vida de los estudiantes.

La competencia genérica número cinco de la Reforma Integral de la Educación Media Superior (RIEMS) (2008: 3-4) en su acuerdo 444, señala: que el estudiante piensa crítica y reflexivamente y describe atributos relacionados con el método científico como son la construcción de hipótesis, el diseño y la experimentación, para llegar a conclusiones y comunicar la información. Lo anterior forma parte del Currículo por Competencias 2015 del Centro de Educación Media (CEM). Por otra parte, en el Modelo Educativo Institucional (MEI) (UAA, 2015: 6), se puede identificar en el componente de la formación intelectual del estudiante, que los estudiantes deberán asumir una actitud reflexiva, ética, científica, crítica y selectiva.

El Programa del Bachillerato Internacional (BI) que se imparte en la institución, hace énfasis en los enfoques de enseñanza y aprendizaje que permean el entorno educativo, destacando el enfoque de la enseñanza basada en la indagación. Para el caso de la asignatura de Biología como parte de 
las ciencias experimentales, los estudiantes llevan a cabo una "investigación individual" como experiencia de aprendizaje. Ambos programas están sujetos a evaluación externa como parte fundamental del proceso educativo.

Mediante la propuesta del Aprendizaje Basado en la Indagación (ABI), se pretende valorar la curiosidad natural de los estudiantes y lograr que se pregunten en forma continua las razones de los fenómenos que se presentan en su entorno inmediato, que investiguen y den respuesta a las preguntas planteadas como parte del desarrollo del pensamiento científico.

El programa de educación científica basada en la indagación del Ministerio de Educación de Costa Rica (2009: 7), identifica los aspectos: hacer, pensar y comunicar como procesos articulados tendientes a la formación del pensamiento científico. Para el caso de la experiencia en el contexto del BI, los aspectos se relacionan con competencias para la investigación: pensar (predecir, diseñar, concluir, evaluar), hacer (observar, experimentar, registrar) y comunicar (escribir, expresar).

Otra forma de representar el modelo del proceso del ABI, es el llamado "ciclo de aprendizaje mediante indagación" (Esquema 1), adaptado de Feinsinger (2014), en el que se identifican tres momentos: inda- gación, en donde la pregunta surge del entorno y curiosidad de los jóvenes, la acción o experimentación, que en forma simplificada intenta responder a la(s) pregunta(s) mediante la obtención de datos, y finalmente la reflexión o conclusión, en donde se evalúan los resultados y el proceso de indagación.

La propuesta metodológica hace énfasis en la evaluación de los aprendizajes mediante el "cartel científico" como una tarea compleja, en la que el estudiante comunica el proceso que incluye los tres momentos del ciclo del aprendizaje.

La estrategia de aprendizaje está basada en la "investigación individual", en la que los estudiantes del BI realizan la actividad práctica guiada por el docente en la siguiente secuencia: Planificación: que incluye la inducción y diseño de la investigación (indagación) (1 hr), Acción: experimentación y análisis $(10$ hr), Evaluación: presentación del método experimental y procesamiento de datos para su retroalimentación entre pares $(2 \mathrm{hr})$ y Comunicación: elaboración y presentación del "cartel científico" (2 hr).

En la experiencia de las investigaciones realizadas por estudiantes en la asignatura de Biología durante el quinto semestre (2015) del BI, se pueden identificar los temas seleccionados por los estudiantes como "análisis del pH", "observación de

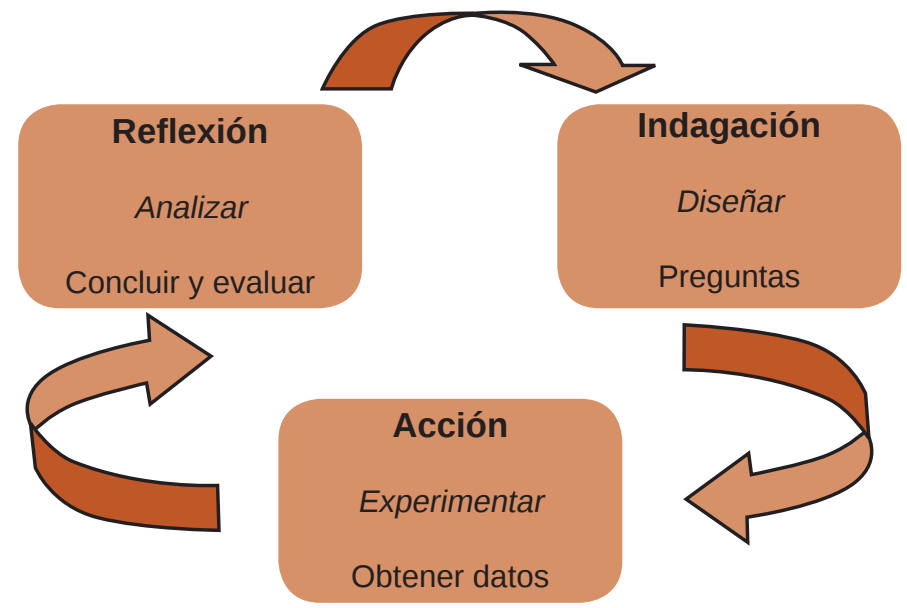

Esquema 1. Modelo del proceso del ABI, elaborado por Francisco Acosta Collazo (2016), a partir del "Ciclo de aprendizaje mediante indagación", propuesto por Feinsinger (2014). 
ADN", "crecimiento bacteriano", "descomposición de fruta", "calidad del agua", "actividad enzimática”, etc. (Gráfica 1); sin embargo, su mayoría (33.3 $\%$ ) se centra en el tema de germinación, lo que sugiere buscar estrategias para que los estudiantes tengan un panorama más amplio en las prácticas experimentales. A grandes rasgos, en la experiencia se pueden reconocer las siguientes fortalezas y áreas de oportunidad como parte del proceso de indagación:

- Indagación: las investigaciones realizadas presentan información de referencia adecuada y denotan interés y creatividad; sin embargo, la fase de pregunta de investigación y definición de las variables representó cierta dificultad para algunos de los estudiantes.

- Acción: los jóvenes evidencian habilidades para el manejo y presentación de datos en hojas de cálculo. Algunas investigaciones presentan datos insuficientes para el procesamiento estadístico.

- Reflexión: la mayor parte de los trabajos presentan conclusiones fundamentadas en los resultados, la fase de coevaluación fue enriquecedora para los estudiantes. Se requiere trabajar en la descripción de limitantes y fuentes de error en las investigaciones.

En las ciencias experimentales, es necesario promover la cultura científica más que la transmisión del conocimiento, se puede lograr que los jóvenes vinculen sus intereses y habilidades con la problemática de su entorno. Rivera y col. (2012) presentan herramientas prácticas que facilitan el desarrollo de habilidades y competencias para la investigación.

El enfoque de la formación basada en competencias del CEM, promueve la formación del pensamiento crítico en forma explícita, por lo que es necesario cambiar el papel de docente pasivo a docente activo centrado en fomentar las competencias y habilidades para la investigación. Es claro que el pensamiento científico requiere de movilización de saberes, lo cual se puede observar en el "cartel científico" utilizado como instrumento de evaluación en la propuesta metodológica.

De igual manera, el ABI, como parte de los enfoques de la enseñanza que promueve el BI, fo-
Temas de investigación individual

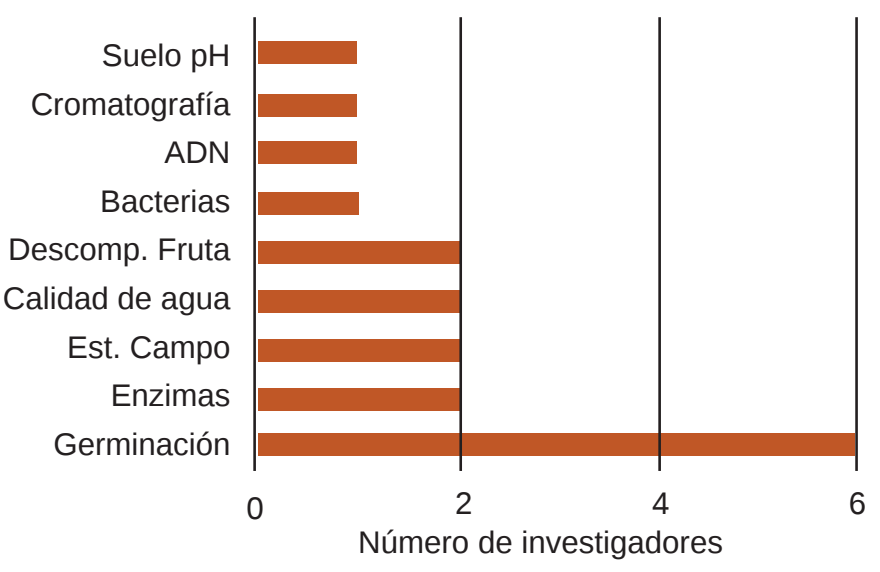

Gráfica 1. Temas de investigación, elaborada por Francisco Acosta Collazo (2016), a partir de las investigaciones realizadas por los estudiantes del quinto semestre del BI, en la asignatura de Biología (2015).

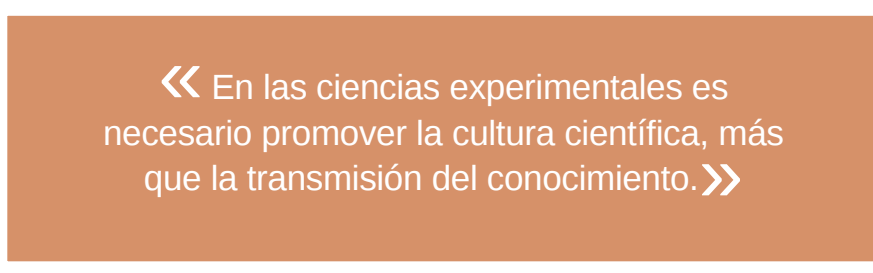

menta los cuestionamientos y responsabilidad de los estudiantes en el procesamiento de la información, para llegar a la reflexión como un paso importante en el ciclo de indagación. Los resultados de la experiencia son parte del reto de transformar la cotidianeidad del aula a un entorno atractivo y relevante para los jóvenes.

Puede notarse que la experimentación (acción) está orientada para utilizar los recursos del entorno de los jóvenes, pues no siempre se requiere del laboratorio escolar formal para la aplicación del método científico, además, el aprendizaje se vincula a disciplinas del currículo escolar, por ejemplo, la aplicación de técnicas estadísticas básicas o los trabajos de monografía que son parte del programa del BI.

Los esfuerzos de la comunidad educativa del CEM en torno a los programas educativos, se ven reflejados en las evaluaciones externas como lo son el Consejo para la Evaluación de la Educación del tipo Medio Superior (COPEMs) y la Organización del Bachillerato Internacional (OBI), ante los cuales se obtienen buenos resultados. 

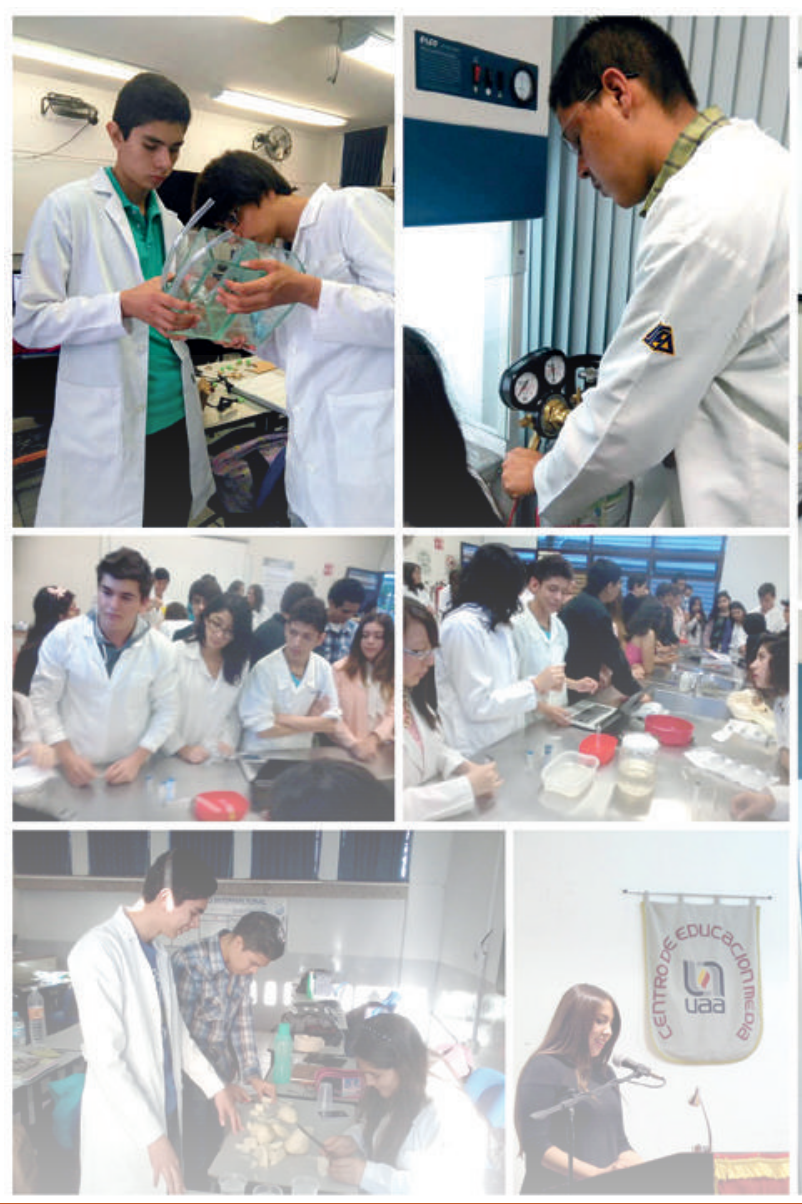

Fuentes de consulta

Feinsinger, P. (2014). El Ciclo de Indagación: una metodología para la investigación ecológica aplicada y básica en los sitios de estudios socio-ecológicos a largo plazo, y más allá. Bosque (Valdivia), 35(3), 449-457. Recuperado el 12 de junio de 2016, en: http://bit. ly/2fyV7eG.

Gutiérrez, V. (1998). Actitudes de los estudiantes hacia la ciencia. México: UAA.

Ministerio de Educación de Costa Rica (2009). La indagación en la enseñanza de las ciencias. Módulo 1. MEP. Recuperado el 13 de junio de 2016, en: http://www.mep.go.cr/sites/ default/files/recursos/archivo/modulo_ciencias1.pdf.

Organización de las Naciones Unidas para la Educación, la Ciencia y la Cultura. (2005). ¿Cómo promover el interés por la cultura científica? Chile: UNESCO-ORLEAC. Recuperado el 28 de junio de 2016, en: http://unesdoc.unesco.org/ images/0013/001390/139003s.pdf.
Rivera, M., Arango, L., Torres, C., Salgado, R., García, F. y Caña, L. (2012). Competencias para la investigación. México: Trillas.

Secretaría de Educación Pública. (2008). Acuerdo número 444 por el que se establecen las competencias que constituyen el marco curricular común del Sistema Nacional de Bachillerato. En Diario Oficial, primera sección, 21 de octubre de 2008. México: SEPRIEMS. Recuperado el 28 de junio de 2016, en: http://bit.ly/2ffK8ZD.

Universidad Autónoma de Aguascalientes. (2015). Bachillerato General. Currículum por Competencias 2015. México: UAA-CEM. Recuperado el 28 de junio de 2016, en: http://www. uaa.mx/centros/cem/.

Universidad Autónoma de Aguascalientes. (2015). Modelo Educativo Institucional, en Correo Universitario, No. 15, séptima época, 29 de mayo de 2015. Primera reimpresión. México: UAA. Recuperado el 28 de junio de 2016, en: http://www.uaa.mx/direcciones/dgdp/defaa/. 